\title{
Fundus changes in (type II) mesangiocapillary glomerulonephritis simulating drusen: a histopathological report
}

\author{
JOSEPHINE DUVALL-YOUNG, ${ }^{1}$ MARY K MACDONALD, ${ }^{2}$ \\ AND NICOL M MCKECHNIE
}

From the ${ }^{1}$ Princess Alexandra Eye Pavilion, Royal Infirmary, Edinburgh; ${ }^{2}$ Department of Pathology, University of Edinburgh; and ${ }^{3}$ Department of Pathology, Institute of Ophthalmology, London

SUMMARY We report for the first time to our knowledge the histopathological findings in the eye of a patient with type II mesangiocapillary glomerulonephritis (dense deposit disease) in which a deposit of material morphologically very similar to that which is pathognomonic for the disease in the kidney was demonstrated in Bruch's membrane. The nature of the deposit in the renal lesion is unknown but is considered to represent a structural alteration secondary to a reaction with anticomplement antibody. Clinically the fundus appearance resembled that seen in drusen.

Glomerulonephritis can be classified according to the histopathological appearances. In type II mesangiocapillary (MCGN type II) glomerulonephritis a deposit, characteristically electron dense on electron microscopy, is seen in the glomerular capillary basement membranes. We report here the clinical case and ocular histopathological findings in a patient with MCGN type II in whom a deposit of abnormal material in the choroid and Bruch's membrane similar to that demonstrated in the glomerulus was present. The choroid and the kidney have anatomical similarities in that they both have a large capillary bed made up of fenestrated vessels, and we suggest that the two sites may be affected by similar disease processes.

\section{Case report}

\section{CLINICAL HISTORY}

An18-year-old male patient presented in March 1981 with a two-week history of malaise, leg cramps, ankle swelling, and nocturia. On renal biopsy his nephrotic syndrome was found to be due to type II mesangiocapillary glomerulonephritis. He was initially treated with diuretics, a low salt diet, and hydrallazine to control his blood pressure, which was raised to $150 /$ $100 \mathrm{mmHg}$ at presentation. His renal function,

Correspondence to J Duvall-Young, FRCS Ed, Department of Ophthalmology, Manchester Royal Eye Hospital, Oxford Road, Manchester M13 9WH. however, continued to deteriorate, and in July he was started on plasmapheresis and immunosuppressive treatment. Having been normotensive since his initial presentation he became hypertensive in September with a blood pressure of $180 / 120 \mathrm{mmHg}$ supine, $120 / 100 \mathrm{mmHg}$ erect. The blood pressure was controlled within a few days with prazosin and metoprolol. However, during that time he complained of blurred vision and was found to have corrected visual acuities of $6 / 24$ in each eye. The anterior segments were normal. He had bilateral papilloedema, macular oedema, and scattered flameshaped haemorrhages. Over the next month the vision in the right eye deteriorated to hand movements only, while in the left it improved to $6 / 12$ with gradual resolution of the signs. At about this time he was started on haemodialysis.

On 9 July 1982 he returned to the eye department with a complaint of pain in the right eye which occurred only while on dialysis. In the right eye there was no perception of light, an afferent pupil defect, corneal oedema, and an intraocular pressure of 52 $\mathrm{mmHg}$. The left eye had a visual acuity of $6 / 9$, with no fundus abnormalities. The patient was treated with topical steroids, atropine $1 \%$ and timolol $0.5 \%$, but he continued to complain of severe intermittent pain while on dialysis. On 27 July when seen again he was found to have a massive exudative bullous retinal detachment. The intraocular pressure remained raised, and he was admitted to hospital for attempted 


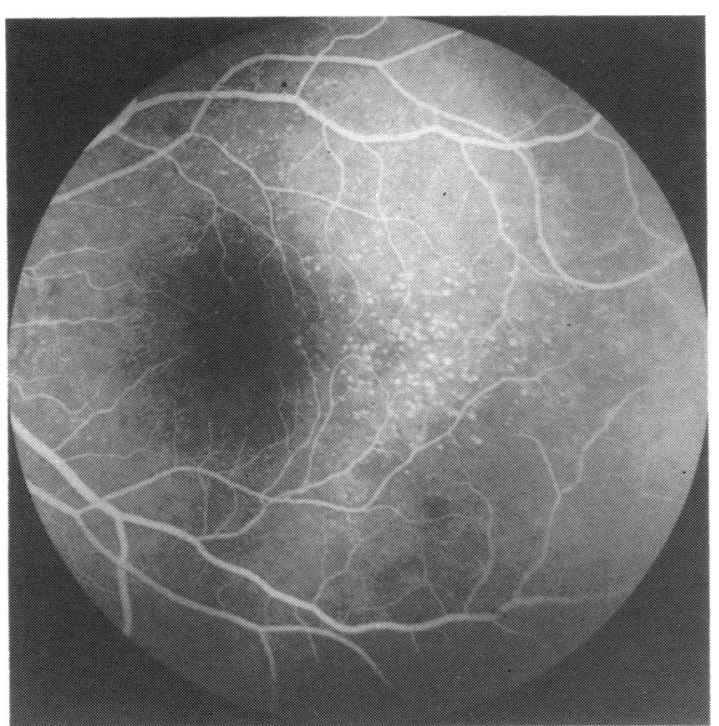

Fig. 1 Fluorescein angiogram of left eye showing multiple hyperfluorescent foci with the characteristics of drusen.

medical control with the addition of acetazolamide. This failed, as did two retrobulbar injections of alcohol, to alleviate his pain, and the right eye was enucleated on 10 August 1982. Fluorescein angiograms of the left eye on 6 September showed no disturbance of choroidal or retinal circulation. Although the fundus appeared normal, there were numerous hyperfluorescent discrete foci at the posterior pole and concentrated in the perifoveal area (Fig. 1), fluorescing early in the angiogram, and becoming more intensely fluorescent throughout. These are the angiographic appearances of drusen.

The patient had remained well on continuing renal dialysis with no change in the appearance of the left eye, but he collapsed and died on 9 April 1983. Necropsy revealed no other cause of death and it was concluded that he had had a cardiac arrest or arrhythmia. Examination of the kidneys yielded no additional information. The left eye was not obtained for examination.

\section{RENAL BIOPSY PATHOLOGY}

Light microscopy. There were eleven glomeruli in the biopsy. All the tufts showed the characteristic appearances of mesangiocapillary glomerulonephritis with marked mesangial increase, involving particularly the matrix, but also some cellular proliferation. Long stretches of the capillary basement membrane stained highly periodic acid Schiff (PAS)positive and thickened. Five of the glomeruli showed large epithelial crescents.
Immunofluorescence microscopy showed large amounts of $\mathrm{C} 3$ and IgM in capillary walls, the latter in focal and segmental distribution. Little positive fluorescence was seen in the mesangium. $\mathrm{C} 3$ and $\mathrm{C} 4$ were present also in some tubular basement membranes.

Transmission electron microscopy showed the presence of an electron dense deposit throughout the glomerular capillary basement membrane, thickening the membrane irregularly in some places (Fig. 2).

The appearances were those characteristic of type II mesangiocapillary glomerulonephritis (dense deposit disease).

\section{EYE PATHOLOGY}

Macroscopic examination and preparation of tissue. The right globe was immediately fixed in $10 \%$ formol saline. The eye was opened horizontally, revealing a total retinal detachment with a subretinal coagulum.

The pupil-optic nerve block was processed through paraffin, and sections were examined after staining with the following: haematoxylin and eosin, periodic acid Schiff, Bodian, Loyez, and Martius scarlet blue. Immunoperoxidase staining for IgG, IgM, kappa chain, lambda chain, C3 and C4 was also undertaken.

Tissue from the calottes was prepared for transmission electron microscopy.

Sections of 1 to $2 \mu \mathrm{m}$ were cut and stained with toluidine blue for orientation by light microscopy. Sections from selected blocks were cut at 50 to $80 \mathrm{~nm}$, stained with uranyl acetate and lead citrate, and examined and photographed with a Phillips 301 electron microscope.

Light microscopic examination. The corneal epithelium was thinned, with intraepithelial oedema and some bullous separation. The drainage angle was closed by an extensive neovascular membrane over the iris surface, with considerable ectropion uveae and a mild mixed inflammatory cell infiltrate in the iris. The ciliary processes were atrophic, with PASpositive deposit in the basement membrane and the stroma of the processes.

The choroid was thinned, but the vessels in all layers were patent (Fig. 3). There was an extensive deposit of PAS-positive material both in the walls of the choroidal blood vessels and in Bruch's membrane, which showed both linear thickening (Fig. 3) and focal deposition, resembling drusen; this was present throughout but most marked at the posterior pole. The deposit stained positively for fibrin with Martius scarlet blue, unlike typical drusen. No immunoglobulin or complement could be demonstrated in the deposits.

The retinal pigment epithelium showed some proliferation, with the formation of a cellular membrane inside the pigment epithelium. There was a 


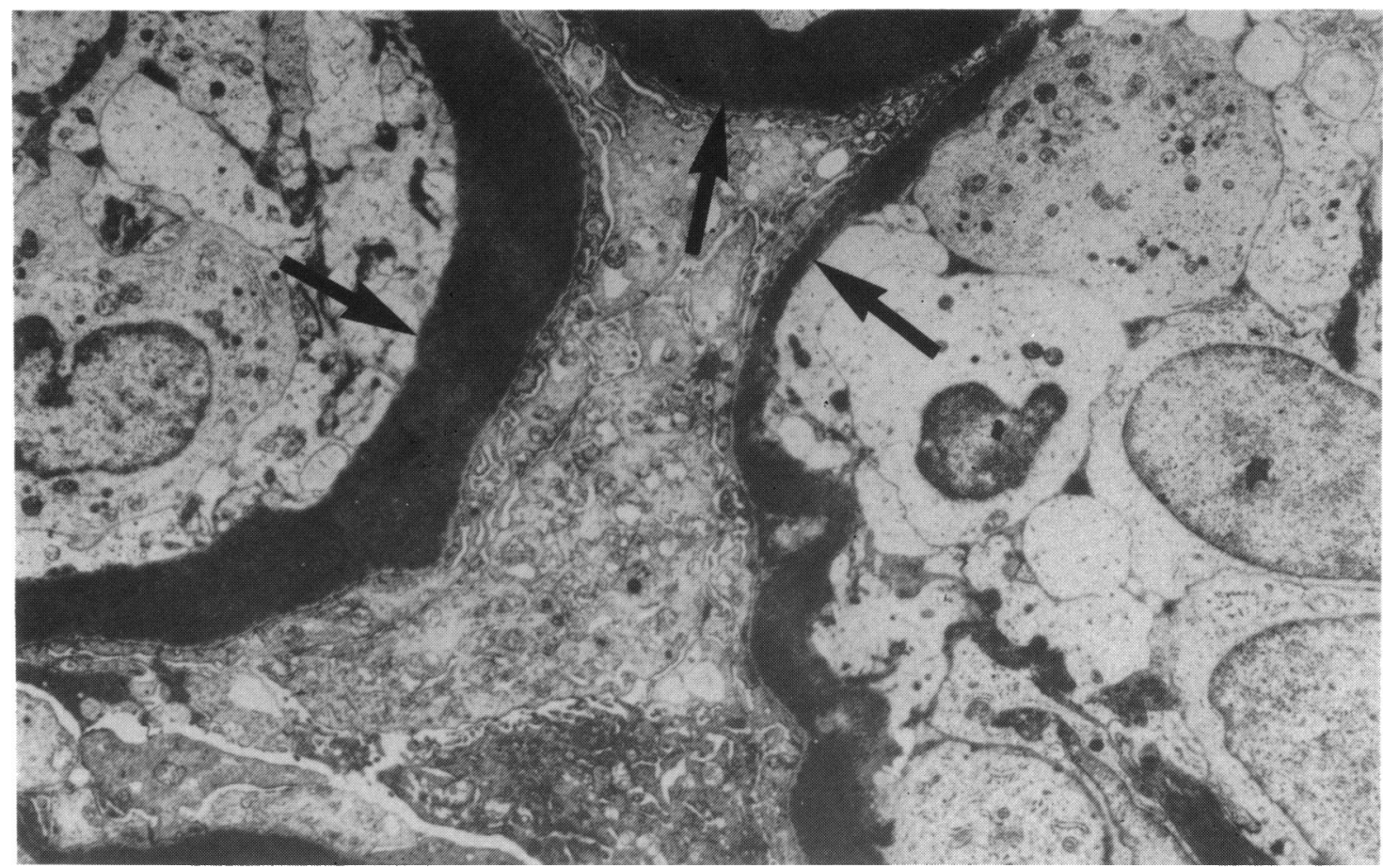

Fig. 2 Electron micrograph of renal glomerulus. The basement membrane of the walls of three glomerular capillaries can be seen (arrows); the membrane varies in width and is abnormally electron dense. $\times 2000$.

total retinal detachment, with extensive recent and organising intraretinal haemorrhage. The retinal architecture was completely replaced by glial cells, and the subretinal space was filled with eosinophilic material showing cholesterol clefts and containing many pigment laden macrophages. Some of the retinal vessels, particularly at the periphery, showed thickening of their walls. There was an extensive fibrovascular preretinal membrane.
The optic nerve was markedly atrophic with very few nerve axons, and showed anterior demyelination. The posterior ciliary vessels were patent but fibrin was focally deposited in their walls.

Electron microscopy. It was confirmed that a deposit was present in the basement membrane of the ciliary epithelium (Fig. 4) and throughout the extent of the inner collagenous layer of Bruch's membrane, where it took two forms, being both linear and focal



Fig. 3 Choroid and Bruch's membrane showing both focal (f) and linear (1) thickening. Note that the vessels in all layers of the choroid are patent, but show some deposition of material in their walls (arrow). $\times 1000$. 




Fig. 4 Electron micrograph of ciliary processes showing dense deposit (d) in the basement membrane. $\times 1500$. Inset $\times 6000$. Note also deposit in basement membrane of choriocapillaris.

(Fig. 5). The deposit was present also in capillary basement membranes. The material was electron dense and amorphous, as in the kidney, though less densely staining.

\section{Discussion}

Type II mesangiocapillary glomerulonephritis is a relatively rare but well recognised condition. The histopathological hallmark is the presence of a linear electron dense deposit in the glomerular capillary basement membrane. ${ }^{\prime}$ The pathogenesis is unknown, but there is evidence that an anticomplement antibody is implicated, and the serum complement is characteristically low. However, the situation is complex, and as yet there is no definite knowledge of the nature of the dense material in the basement membrane, though it has been suggested that it represents alteration in a glycoprotein of the basement membrane itself. Immunopathology often shows $\mathrm{C} 3$ almost alone in the capillary walls, as in this case.

In a previous experimental study of the choriocapillaris a comparison between the anatomy of the glomerulus and of the choriocapillaris/Bruch's membrane/retinal pigment epithelial complex has been drawn. ${ }^{2}$ To our knowledge this is the first case to be reported of type II MCGN with clinical and histopathological evidence of ocular involvement similar to the glomerular lesion.

The results of previous studies of ocular pathology coexisting with glomerular disease are shown in Table 1.

In a mouse model of systemic lupus erythematosus $(\mathrm{SLE})^{3}$ the immunoglobulin deposits identified were found in the choroidal vessels, Bruch's membrane, and ciliary processes as well as in glomeruli, the choroid plexus, and the capillaries at the epidermaldermal junction. A similar distribution of deposition of immune complexes is found in rat serum sickness. ${ }^{4}$ 


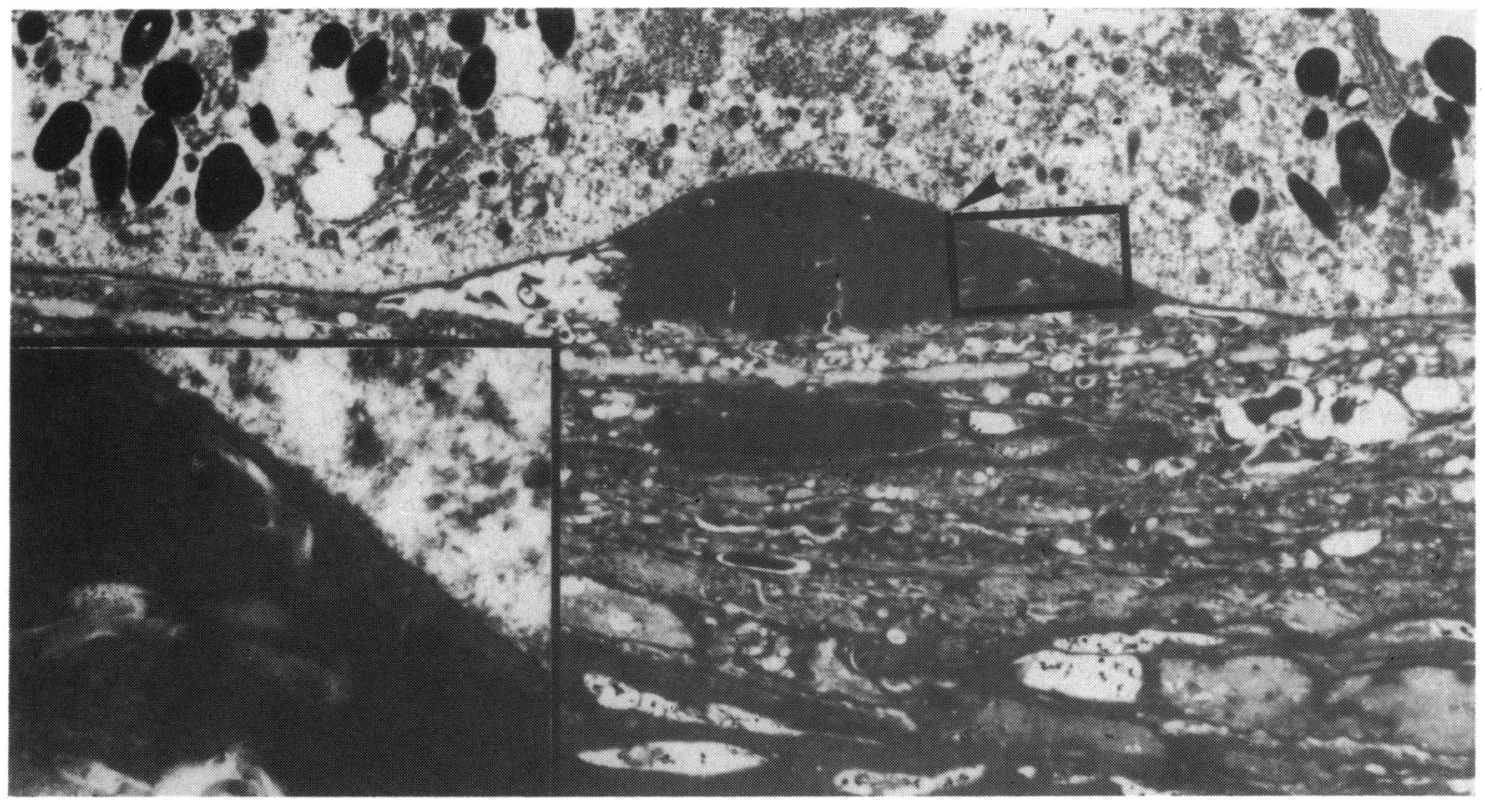

Fig. 5 Electron micrograph of Bruch's membrane showing dense deposit in drusen-like distribution and also in the chorocapillaris (arrow) $\times 2730$. Inset: at higher power the deposit is homogeneously dense. $\times 20600$.

However, there are only two reports of deposition of immune complexes in human cases, one describing the post-mortem findings in a case of SLE in which the deposits were not detectable clinically, ${ }^{5}$ and the other a case of Goodpasture's syndrome (antiglomerular basement membrane disease) in which immune complexes were deposited in a uniform linear pattern due to an antigen/antibody reaction in the basement membrane. ${ }^{6}$ Drusen-like deposits have been described clinically in a kappa chain monoclonal gammopathy ${ }^{7}$ and occur in $21 \%$ of patients with SLE. ${ }^{8}$

We presume that in the case we report abnormal material, possibly of immune complex nature, has been derived from the blood, having escaped through damaged vessel walls. Aronson et al. ${ }^{5}$ found in their patients with SLE, who were not hypertensive, that there was leakage of fluorescein from atypical drusen into the subretinal fluid under areas of localised retinal detachment.

We would suggest that abnormal deposits in Bruch's membrane and the choroid may be not infrequent in immunologically mediated renal disease, but that they remain unrecognised because the eyes have not been examined histologically.

The deposit in the eye did not stain for immunoglobulin or complement. However, the dense deposit typical of the lesion is not itself immunoglobin or complement but rather represents a structural alteration in extracellular tissue which may be a result of deposition of immunoglobin or complement. The immunoglobulin or complement may be only transiently associated with the basement membrane, which, however, remains morphologically changed. The failure to demonstrate immunoglobulin or complement in the ocular lesion in this case, and in

Table 1 Summary of literature

\begin{tabular}{|c|c|c|c|c|c|}
\hline & \multirow[t]{2}{*}{ Disease state } & \multicolumn{4}{|c|}{ Site of deposition } \\
\hline & & Conjunctiva & Ciliary body & $\begin{array}{l}\text { Bruch's } \\
\text { membrane }\end{array}$ & $\begin{array}{l}\text { Choroidal vascular } \\
\text { basement membrane }\end{array}$ \\
\hline Jampol et al. ${ }^{6}$ & Goodpasture's disease & - & + & ++ & ++ \\
\hline Pertschuk et al. ${ }^{3}$ & Animal model: SLE & - & ++ & ++ & ++ \\
\hline Peress et al. ${ }^{+}$ & Animal model: rat, chronic serum sickness & - & ++ & - & - \\
\hline Aronson et al. ${ }^{5}$ & SLE & + & ++ & ++ & ++ \\
\hline Nik et al. ${ }^{\prime}$ & IgA-kappa chain monoclonal gammopathy (no histology) & - & - & + & - \\
\hline
\end{tabular}


the glomerular lesion in some other cases, may be related to the timing of the histological examination in relation to the onset or activity of the disease.

Our patient had a retinal venous occlusion and a retinal detachment, both attributable to accelerated hypertension. Retinal detachment is a well described complication of accelerated-phase hypertension, particularly in toxaemia of pregnancy. ${ }^{9}$ The cause is thought to be massive exudation of fluid into the choroid with breakdown of the blood-eye barrier. ${ }^{10}$ This has been demonstrated by fluorescein angiography in which dye is shown to leak out from the choroid into the subretinal fluid. ${ }^{11}$ In all the reported cases of retinal detachment associated with renal failure the patients had marked hypertension. ${ }^{13}{ }^{14}$ The vascular changes in the choriocapillaris could be attributed to hypertension in this case,,$^{15} 16$ but a deposit such as one described in the stroma of the choriocapillaris and Bruch's membrane has never been previously described. Additional evidence to support our belief that we are describing a new phenomenon is that we did not see typical infarcts of the pigment epithelium, ${ }^{10}$ though these are not an invariable feature of accelerated hypertensive retinopathy.

Retinal venous occlusion may occur in patients on renal dialysis, probably as a result of raised intraocular pressure. ${ }^{17}{ }^{18}$ The relative hyperosmolality of the intraocular fluid after dialysis results in an influx of water into the eye under osmotic pressure, with a rise of intraocular pressure of up to $16 \mathrm{mmHg}{ }^{18}$ This phenomenon could also account for the pain of which this patient complained following episodes of dialysis.

This case demonstrates involvement of the choriocapillaris and Bruch's membrane and of the renal glomeruli in a similar morphological change; a clinical correlation with drusen-like spots in the fundus is also present. The choriocapillaris/Bruch's membrane/RPE complex has morphological similarities with the glomerulus. We suggest that both sites may be affected by similar disease processes and that the response of the choroid in renal disease merits further investigation.
We thank Professor W R Lee, University of Glasgow, for his advice and encouragement, and we are grateful to $\operatorname{Dr} A$ Lambie and $D r R J$ Winney, Medical Renal Unit, Royal Infirmary, Edinburgh, for permission to report this case.

\section{References}

1 Burkholder PM, Hyman CR, Kryer RP. Characterisation of mixed membranous and proliferative glomerulonephritis. In: Kincaid-Smith P, Matthew TH, Becker EL, eds. Glomerulonephritis. New York: J Wiley, 1973: 557.

2 Duvall J, Tso MOM. Cellular mechanisms of resolution of drusen after laser coagulation. An experimental study. Arch Ophthalmol 1985; 103: 694-703.

3 Pertschuk LP, Szabo K, Vuletin JC, Rainford EA. Ocular immunopathology in murine lupus. Arch Pathol Lab Med 1977; 101: $122-4$.

4 Peress NS, Miller.F, Palu N. The immunopathological effects of chronic serum sickness in rat choroid plexus, ciliary processes and renal glomeruli. J Neuropathol Exp Neurol 1977; 36: 726-33.

5 Aronson AJ, Ordonez NG, Diddie KR, Ernest T. Immune complex deposition in the eye in systemic lupus erythematosus. Arch Intern Med 1979; 139: 1312-3.

6 Jampol LM, Lahan M, Albert DM, Craft J. Ocular clinical findings and basement membrane changes in Goodpasture's syndrome. Am J Ophthalmol 1975; 79: 452-63.

7 Nik NA, Martin NF, Berler DK. Corneal crystalline deposits and drusenosis associated with IgA kappa chain monoclonal gammopathy. Ann Ophthalmol 1985; 17: 303-7.

8 Gold DH, Morris DA, Henkind P. Ocular findings in systemic lupus erythematosus. $\mathrm{Br}$ J Ophthalmol 1972; 56: 800-4.

9 Fry WE. Extensive bilateral retinal detachment in eclampsia, with complete reattachment. Arch Ophthalmol 1929; 1: 609-14.

10 Klein BA. Ischaemic infarcts of the choroid. Am J Ophthalmol 1969; 66: 1069-74.

1 Gitter KA, Houser BP, Sarin LK, Justice J. Toxemia of pregnancy. Arch Ophthalmol 1968; 80: 449-54.

12 Fastenberg DM, Fetkenhour LL, Chorornokos E, Shock DE. Choroidal vascular changes in toxemia of pregnancy. Am J Ophthalmol 1980; 89: 362-8.

13 Buchanan WS, Elliss PP. Retinal separation in chronic glomerulonephritis. Arch Ophthalmol 1964; 71: 182-6.

14 Ellis PP, Fonkin HA. Retinopathies of chronic glomerulonephritis. Arch Ophthalmol 1966; 75: 36-41.

15 Ashton N. The eye in malignant hypertension. Trans Am Ophthalmol Soc 1970; 76: 17-40.

16 Kishi S, Tso MOM, Hayreh SS. Fundus lesions in malignant hypertension I. A pathologic study of experimental hypertensive choroidopathy. Arch Ophthalmol 1985; 103: 1189-97.

17 Pratt NV, Devenelia G. Central retinal vein occlusion following peritoneal dialysis. Am J Ophthalmol 1970; 70: 337-40.

18 Ramsell JT, Ellis PP, Paterson CA. Intraocular pressure changes during haemodialysis. Am J Ophthàlmol 1971; 72: 926-30.

Accepted for publication 12 May 1988. 